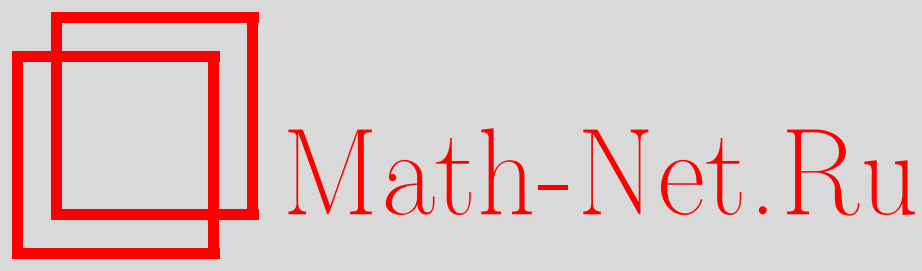

А. В. Куров, Г. А. Сарданашвили, Глобально суперинтегрируемые гамильтоновы системы, ТМФ, 2017, том 191, номер 3, 389-406

DOI: https://doi.org/10.4213/tmf9225

Использование Общероссийского математического портала Math-Net.Ru подразумевает, что вы прочитали и согласны с пользовательским соглашением http://www . mathnet.ru/rus/agreement

Параметры загрузки:

IP : 54.224 .60 .19

26 апреля 2023 г., 15:24:06

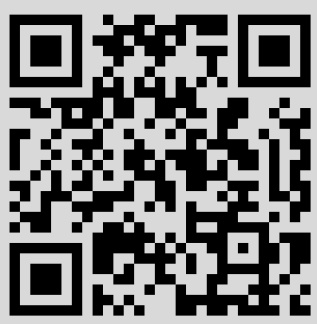




\title{
ФИЗИКА
}

Том 191, № 3

июнь, 2017

(С) 2017 г. $\quad$ А. В. Куров*, Г.А. Сарданашвили $*$

\section{ГЛОБАЛЬНО СУПЕРИНТЕГРИРУЕМЫЕ ГАМИЛЬТОНОВЫ СИСТЕМЫ}

\begin{abstract}
Обобщение теоремы Мищенко-Фоменко для симплектических суперинтегрируемых систем на случай произвольного, не обязательно компактного, инвариантного подмногообразия позволяет дать глобальное описание суперинтегрируемой гамильтоновой системы, которая может распадаться на несколько неэквивалентных глобально суперинтегрируемых систем на непересекающихся открытых подмножествах фазового симплектического многообразия, имеющих как компактные, так и некомпактные инвариантные подмногообразия. Характерным примером такой композиции глобально суперинтегрируемых систем является движение в центрально-симметричном поле, в частности двумерная задача Кеплера.
\end{abstract}

Ключевые слова: вполне интегрируемая система, суперинтегрируемая система, переменные действие-угол, центрально-симметричный потенциал, система Кеплера.

DOI: https://doi.org/10.4213/tmf9225

\section{1. ВВЕДЕНИЕ}

Пусть $(Z, \Omega)-2 n$-мерное связное симплектическое многообразие и

$$
F=\left(F_{1}, \ldots, F_{k}\right), \quad n \leqslant k<2 n,
$$

- суперинтегрируемая (т. е. некоммутативная вполне интегрируемая) система производящих функций на многообразии $(Z, \Omega)$ (см. ниже определение 1$)$. Известная теорема Мищенко-Фоменко (см. ниже теорему 2) устанавливает, что в окрестности компактного инвариантного подмногообразия такой системы могут быть введены обобщенные переменные действие-угол [1]-[3]. Если $k=n$, это случай вполне интегрируемых систем (см. ниже определение 2) и теоремы Лиувилля-Арнольда [4], [5].

Вслед за теоремой Лиувилля-Арнольда для вполне интегрируемых систем и теоремой Пуанкаре-Ляпунова-Нехорошева для частично интегрируемых систем [6], [7] теорема Мищенко-Фоменко была обобщена на суперинтегрируемые системы с произвольными, т. е. не обязательно компактными, инвариантными подмногообразиями

* Московский государственный университет им. М. В. Ломоносова, Москва, Россия. E-mail: kurov.aleksandr@physics.msu.ru 
(см. ниже теорему 1) [8]-[10]. Эти подмногообразия диффеоморфны тороидальным цилиндрам

$$
\mathbb{R}^{m-r} \times T^{r}, \quad m=2 n-k, \quad 0 \leqslant r \leqslant m .
$$

В частности, теорема Мищенко-Фоменко (теорема 2) описывает суперинтегрируемые системы, производящие функции (1) которых образуют только компактную алгебру Ли (см. ниже (9)). Группа потоков их гамильтоновых векторных полей компактна. Так как компактное расслоение имеет компактные слои, то инвариантные подмногообразия такой суперинтегрируемой системы компактны. Упомянутая выше теорема 1 применима к суперинтегрируемым системам с произвольной алгеброй Ли производящих функций (раздел 5). Это позволяет дать описание суперинтегрируемых систем, которые могут распадаться на подсистемы как с компактными, так и с некомпактными алгебрами Ли производящих функций. Характерным примером такой суперинтегрируемой системы служит двумерная задача Кеплера (раздел 7).

В разделе 3 представлено расширение теоремы 1 на случай глобально суперинтегрируемых систем (определение 3), которые допускают глобальные обобщенные переменные действие-угол (теоремы 4 и 6). Теорема 4 устанавливает условия существования таких глобальных переменных (см. [11] для случая вполне интегрируемых систем и [3], [12], [13] для суперинтегрируемых систем с компактными инвариантными подмногообразиями). Эти условия в общем случае не выполняются на всем фазовом симплектическом пространстве, и суперинтегрируемая система может распадаться на несколько неэквивалентных (с разными инвариантными подмногообразиями) глобально суперинтегрируемых систем на непересекающихся открытых подмногообразиях фазового симплектического многообразия [9], [10].

Имея в виду приложения, мы рассматриваем суперинтегрируемые гамильтоновы системы (определение 4), когда гамильтониан системы функционально зависим с ее производящими функциями, которые находятся с ним в инволюции и являются интегралами движения (раздел 4). Тогда гамильтониан такой системы выражается только через обобщенные переменные действия, а движение происходит только по обобщенным угловым переменным (теорема 7).

В качестве характерных примеров суперинтегрируемых гамильтоновых систем, имеющих как компактные, так и некомпактные инвариантные подмногообразия, мы рассматриваем движение в центрально-симметричном поле общего вида в пространствах разной размерности (раздел 6) и, в частности двумерную задачу Кеплера (раздел 7).

\section{2. СУПЕРИНТЕГРИРУЕМЫЕ СИСТЕМЫ}

Мы рассматриваем суперинтегрируемые системы на симплектических многообразиях.

ОПрЕДЕЛЕНИЕ 1 . Пусть $(Z, \Omega)-2 n$-мерное связное симплектическое многообразие с симплектической формой $\Omega$ и $\left(C^{\infty}(Z),\{\cdot, \cdot\}\right)$ - пуассонова алгебра гладких вещественных функций на $Z$. Подмножество $F(1)$ пуассоновой алгебры $C^{\infty}(Z)$ называется суперинтегрируемой системой, если выполняются следующие условия.

1. Все функции $F_{i}$ (называемые производящими функииями суперинтегрируемой системы) независимы, т. е. $k$-форма $\stackrel{k}{\wedge} d F_{i}$ нигде на многообразии $Z$ не равна нулю. 
Следовательно, отображение

$$
\widehat{F}: Z \rightarrow N=\underset{i}{\times} F_{i}(Z) \subset \mathbb{R}^{k}
$$

является субмерсией, т. е. $Z$ - это расслоенное многообразие над областью (стягиваемым открытым подмножеством) $N \subset \mathbb{R}^{k}$ с координатами $\left(x_{i}\right)$ такими, что $x_{i} \circ \widehat{F}=F_{i}$.

2 . На многообразии $N$ существуют гладкие вещественные функции $s_{i j}$ такие, что скобки Пуассона функций $F_{i}$ удовлетворяют соотношениям

$$
\left\{F_{i}, F_{j}\right\}=s_{i j} \circ F, \quad i, j=1, \ldots, k .
$$

3. Матричная функция $\mathbf{s}$ с элементами $s_{i j}(4)$ имеет постоянный коранг $m=2 n-k$ во всех точках $N$.

Если $k>n$, то матрица $\mathbf{s}$ заведомо ненулевая. Если $k=n$, тогда $\mathbf{s}=0$. Это случай вполне интегрируемой системы.

ОПРЕДЕЛЕНИЕ 2. Подмножество $F(1), k=n$, пуассоновой алгебры $C^{\infty}(Z)$ на симплектическом многообразии $(Z, \Omega)$ называется вполне интегрируемой системой, если $F_{i}$ являются независимыми функциями в инволюции.

Два следующих утверждения проясняют структуру суперинтегрируемых систем [3], [8].

Лемма 1 [14]. Пусть $(Z, \Omega)$ - симплектическое многообразие и $\widehat{F}: Z \rightarrow N(3)-$ расслоенное многообразие, такое что если функции $f, f^{\prime}$ постоянны на слоях $\widehat{F}$, то их скобка Пуассона $\left\{f, f^{\prime}\right\}$ также постоянна. Тогда на $N$ существует единственная коиндуцированная пуассонова структура $\{\cdot, \cdot\}_{N}$ такая, что $\widehat{F}$ - это пуассонов морфизм.

Поскольку любая постоянная на слоях $\widehat{F}(3)$ функция индуцируется некоторой функцией на $N$, то в силу пункта 2 определения 1 суперинтегрируемая система (1) удовлетворяет условию леммы 1 . Поэтому база $N$ расслоенного многообразия (3) наделена коиндуцированной пуассоновой структурой коранга $m$. В координатах $x_{i}$ согласно определению 1 ее бивекторное поле имеет вид

$$
w=s_{i j}\left(x_{k}\right) \partial^{i} \wedge \partial^{j}
$$

Лемма 2 [3]. Пусть задано расслоенное многообразие $\widehat{F}: Z \rightarrow N$ из леммъ 1 со связными слоями. Тогда следующие условия эквивалентны:

1) ранг коиндуцированной пуассоновой структуры на $N$ равен $2 \operatorname{dim} N-\operatorname{dim} Z$;

2) слои $\widehat{F}$ - это интегральные многообразия инволютивного распределения, порождаемого гамильтоновыми векторными полями функиий $\widehat{F}^{*} C$, индуцированных функииями Казимира $C$ пуассоновой структурь (5) на $N$.

Легко убедиться, что расслоенное многообразие $\widehat{F}(3)$ удовлетворяет условию 1 леммы 2 в силу пункта 3 определения 1 , а именно $k-m=2(k-n)$. Его слои называются инвариантными подмногообразиями.

Пусть задана суперинтегрируемая система в соответствии с определением 1. Обобщение теоремы Мищенко-Фоменко на случай некомпактных инвариантных подмногообразий устанавливает следующая теорема [8], [9]. 
Теорема 1. Пусть гамильтоновы векторные поля $\vartheta_{i}$ функиий $F_{i}$ (m.е. $\left.\vartheta_{i}\right\rfloor \Omega=$ $\left.-d F_{i}\right)$ полны, а слои расслоенного многообразия $\widehat{F}(3)$ связны и взаимно дифбеоморфны. Тогда справедливы следующие утверждения.

1. Слои расслоенного многообразия $\widehat{F}(3)$ диффеоморфны тороидалъным иилиндрам (2).

2. Пусть $M$ - слой расслоенного многообразия $\widehat{F}(3)$, тогда существует его открытая насыщенная окрестность $U$, которая является тривиальным главным расслоением со структурной группой (2).

3. Окрестность $U$ имеет расслоенные координаты (обобщенные переменные действие-угол) $\left(I_{\lambda}, p_{s}, q^{s}, y^{\lambda}\right), \lambda=1, \ldots, m, s=1, \ldots, n-m$, такие, что: 1) переменные действия $\left(I_{\lambda}\right)$ - это значения функций Казимира коиндуцированной пуассоновой структуры $\{\cdot, \cdot\}_{N}$ на $\left.\widehat{F}(U) ; 2\right)$ обобщенные угловые переменные $\left(y^{\lambda}\right)$ это координаты на тороидальных цилиндрах; 3) симплектическал форма $\Omega$ на $U$ принимает вид

$$
\Omega=d I_{\lambda} \wedge d y^{\lambda}+d p_{s} \wedge d q^{s}
$$

ЗАмЕЧАниЕ 1. Если полные векторные поля на гладком многообразии образуют базис конечномерной вещественной алгебры Ли, то любой элемент этой алгебры полон [15].

ЗАмечАниЕ 2. Требование полноты гамильтоновых векторных полей $\vartheta_{i}$ производящих функций $F_{i}$ является довольно сильным ограничением. Его можно заменить условием, что гамильтоновы векторные поля функций, индуцированных на $Z$ функциями Казимира на $N$, полны (см. ниже теорему 6).

Если условия теоремы 1 заменить требованием компактности и связности слоев расслоенного многообразия $\widehat{F}(3)$, то мы придем к упомянутой выше теореме Мищенко-Фоменко [1]-[3].

ТеОРема 2. Пусть слои расслоенного многообразия $\widehat{F}(3)$ связны и компактны. Тогда они диффеоморфны тору $T^{m}$ и справедливы утверждения 2, 3 теоремы 1.

ЗАмЕЧАНИЕ 3 . В теореме 2 гамильтоновы векторные поля $\vartheta_{i}$ полны, так как слои расслоенного многообразия $\widehat{F}(3)$ компактны. Известно, что любое векторное поле на компактном многообразии полно.

Если $F(1)$ - вполне интегрируемая система, коиндуцированная пуассонова структура на $N$ является нулевой, и производящие функции $F_{i}$ индуцируются $n$ независимыми функциями на $N$. Тогда теоремы 2 и 1 воспроизводят теорему Лиувилля-Арнольда [4], [5] и ее обобщение (теорема 3) на случай некомпактных инвариантных подмногообразий [6], [7].

Теорема 3. Пусть F (1) - вполне интегрируемая система. Пусть гамильтоновы векторные поля $\vartheta_{i}$ функциц $F_{i}$ полны, а слои расслоенного многообразия $\widehat{F}(3)$ связны и взаимно дифбеоморфны. Тогда утверждения 1 и 2 теоремы 1 справедливы, а утверждение 3 заменяется следующим.

3'. Окрестность $U$ имеет расслоенные координаты (обобщенные переменные действие-угол) $\left(I_{\lambda}, y^{\lambda}\right), \lambda=1, \ldots, n$, такие, что: 1) обобщенные переменные действия $I_{\lambda}$ выражсатся через значения производящих функций $\left.F_{i} ; 2\right)$ обобщенные 
угловые переменные $\left(y^{\lambda}\right)$ - это координаты на тороидальных иилиндрах; 3) симплектическая форма $\Omega$ на $U$ принимает вид

$$
\Omega=d I_{\lambda} \wedge d y^{\lambda} .
$$

Если условия теоремы 3 заменить требованием компактности и связности слоев расслоенного многообразия $\widehat{F}(3)$, то мы придем к теореме Лиувилля-Арнольда [4], [5].

\section{3. ГЛОБАЛЬНО СУПЕРИНТЕГРИРУЕМЫЕ СИСТЕМЫ}

Следует подчеркнуть, что рассматриваемая в обобщенных переменных действие-угол на открытом подрасслоении $U$ расслоенного многообразия $Z \rightarrow N$ (3) суперинтегрируемая система не эквивалентна исходной, так как не существует морфизма пуассоновой алгебры $C^{\infty}(U)$ на $(U, \Omega)$ в алгебру $C^{\infty}(Z)$ на $(Z, \Omega)$. Более того, суперинтегрируемая система на $U$ не обязательно удовлетворяет условиям теоремы 1, так как гамильтоновы векторные поля производящих функций на $U$ могут быть неполными. Поэтому для описания суперинтегрируемых систем посредством обобщенных переменных действие-угол мы введем понятие глобально суперинтегрируемой системы.

ОПРЕДЕЛЕНИЕ 3. Суперинтегрируемая система $F(1)$ на симплектическом многообразии $(Z, \Omega)$ называется глобально суперинтегрируемой, если существуют глобальные обобщенные переменные действие-угол

$$
\left(I_{\lambda}, x^{A}, y^{\lambda}\right), \quad \lambda=1, \ldots, m, \quad A=1, \ldots, 2(n-m),
$$

такие, что: 1) обобщенные переменные действия $\left(I_{\lambda}\right)$ выражаются через значения некоторых функций Казимира $C_{\lambda}$ на пуассоновом многообразии $\left(N,\{\cdot, \cdot\}_{N}\right)$; 2 ) обобщенные угловые переменные $\left(y^{\lambda}\right)$ являются координатами на тороидальном цилиндре $(2) ; 3)$ симплектическая форма $\Omega$ на $Z$ принимает вид

$$
\Omega=d I_{\lambda} \wedge d y^{\lambda}+\Omega_{A B}\left(I_{\mu}, x^{C}\right) d x^{A} \wedge d x^{B} .
$$

Следующая теорема 4 представляет достаточные условия существования глобальных обобщенных переменных действие-угол суперинтегрируемой системы на симплектическом многообразии $(Z, \Omega)[9]-[11]$.

Теорема 4. Суперинтегрируемая система $F$ на симплектическом многообразии $(Z, \Omega)$ является глобально суперинтегрируемой, если выполняются следующие условия:

1) гамильтоновы векторные поля $\vartheta_{i}$ производящих функиий $F_{i}$ полны;

2) расслоенное многообразие $\widehat{F}$ (3) является расслоением со связными слоями;

3 ) коиндуцированная пуассонова структура $\{\cdot, \cdot\}_{N}$ на базе $N$ допускает $m$ независимых функиий Казимира $C_{\lambda}$.

Теорема 4 воспроизводит теорему 1 , если $x^{A}$ - координаты Дарбу коиндуцированной пуассоновой структуры на $N$.

Заметим, что в случае, если инвариантные подмногообразия суперинтегрируемой системы предполагаются связными и компактными, условие 1 теоремы 4 необязательно, так как векторные поля $v_{\lambda}$ на компактных слоях $\widehat{F}$ полны. Условие 2 также выполняется согласно следующей известной теореме [16]. 
Теорема 5. Расслоенное многообразие, слои которого или диффбеоморфнъц $\mathbb{R}^{k}$, или связны и компактны, является расслоением.

В этом случае теорема 4 воспроизводит известный из работы [13] результат.

Если $F$ в теореме 4 - вполне интегрируемая система, то коиндуцированная пуассонова структура на $N$ является нулевой, производящие функции $F_{i}$ индуцированы $n$ независимыми функциями на $N$, а теорема 4 совпадает с теоремой 4 из работы [11].

Заметим, что тот факт, что $N \subset \mathbb{R}^{k}$ является областью, гарантирует, что расслоение $\widehat{F}$ в условии 2 теоремы 4 тривиально, и слои его связны, поскольку многообразие $Z$ связно. Поэтому теорему 4 можно переформулировать следующим образом.

Теорема 6. Суперинтегрируемая система F на симплектическом многообразии $(Z, \Omega)$ глобально суперинтегрируема тогда и только тогда, когда выполняются следующие условия:

1) расслоенное многообразие $\widehat{F}$ (3) является тривиалъным расслоением;

2) коиндуцированная пуассонова структура $\{\cdot, \cdot\}_{N}$ на базе $N$ допускает $m$ независимых функиий Казимира $C_{\lambda}$ таких, что гамильтоновы векторные поля индуиированных ими функииц $\widehat{F}^{*} C_{\lambda}$ на $Z$ полны.

ЗАмечАниЕ 4. Из замечания 1 и условия 2 теоремы 6 следует, что гамильтоново векторное поле функции $\widehat{F}^{*} C$ на $Z$, индуцированной произвольной функцией Казимира $C$ на пуассоново многообразие $N$, полно.

\section{4. СУПЕРИНТЕГРИРУЕМЫЕ ГАМИЛЬТОНОВЫ СИСТЕМЫ}

В автономной симплектической гамильтоновой механике рассматриваются суперинтегрируемые системы, производящие функции которых являются интегралами движения, т. е. они находятся в инволюции с гамильтонианом системы $H$, и функции $\left(H ; F_{1}, \ldots, F_{k}\right)$ всюду функционально зависимы, т. е.

$$
\begin{array}{r}
\left\{H, F_{i}\right\}=0, \\
d H \wedge\left(\stackrel{k}{\wedge} d F_{i}\right)=0 .
\end{array}
$$

ОПредЕЛЕНИЕ 4. Такая система $\left(H ; F_{1}, \ldots, F_{k}\right)$ называется суперинтегрируемой гамильтоновой системой.

Сам гамильтониан, если он регулярен, может входить в число производящих функций суперинтегрируемой гамильтоновой системы. В этом случае условие (8) заведомо выполняется.

Для того чтобы можно было определить эволюцию гамильтоновой системы в любой момент $t \in \mathbb{R}$, предполагают, что гамильтоново векторное поле гамильтониана системы полно. В силу замечания 4 и приведенной ниже леммы 3 гамильтониан суперинтегрируемой системы всегда удовлетворяет этому условию.

Лемма 3. Из равенства (8) следует, что гамильтониан Н постоянен на инвариантных подмногообразиях. Поэтому он индуцирован функцией на многообразии $N$, которая является функиией Казимира пуассоновой структуры (5) в силу условий (7).

Лемма 3 приводит к следующей теореме. 
Теорема 7. Пусть $H$ - гамильтониан глобально суперинтегрируемой гамильтоновой системы с обобщенными переменными действие-угол $\left(I_{\lambda}, x^{A}, y^{\lambda}\right)(6)$. Тогда гамильтониан $H$ зависит только от обобщенных переменных действия $I_{\lambda}$. Следовательно, уравнения движения глобально суперинтегрируемой системы имеют вид

$$
\dot{y}^{\lambda}=\frac{\partial H}{\partial I_{\lambda}}, \quad I_{\lambda}=\text { const }, \quad x^{A}=\text { const. }
$$

Как уже отмечалось, в общем случае фазовое пространство суперинтегрируемой системы может включать несколько непересекающихся открытых подмногообразий, на каждом из которых она является глобально суперинтегрируемой. Однако, поскольку эти подмногообразия не образуют разбиение фазового пространства, они охватывают не все траектории суперинтегрируемой системы, например движение по параболе в задаче Кеплера (см. раздел 7).

\section{5. СУПЕРИНТЕГРИРУЕМЫЕ СИСТЕМЫ НА КОАЛГЕБРАХ ЛИ}

Рассмотрим суперинтегрируемые системы, производящие функции $\left\{F_{1}, \ldots, F_{k}\right\}$ которых образуют $k$-мерную действительную алгебру Ли $\mathcal{G}$ коранга $m$ с коммутационными соотношениями:

$$
\left\{F_{i}, F_{j}\right\}=c_{i j}^{h} F_{h}, \quad c_{i j}^{h}=\text { const. }
$$

Тогда $\widehat{F}(3)$ является отображением моментов многообразия $Z$ в коалгебру Ли $\mathcal{G}^{*}$, наделенную координатами $x_{i}$ в определении 1 [17]. В этом случае коиндуцированная пуассонова структура $\{\cdot, \cdot\}_{N}$ совпадает с канонической структурой Ли-Пуассона на $\mathcal{G}^{*}$, задаваемой пуассоновым бивекторным полем

$$
w=\frac{1}{2} c_{i j}^{k} x_{k} \partial^{i} \wedge \partial^{j}
$$

Ее функции Казимира постоянны на орбитах коприсоединенного действия

$$
\varepsilon_{i}\left(x_{j}\right)=c_{i j}^{k} x_{k}
$$

алгебры Ли $\mathcal{G}$ в $\mathcal{G}^{*}$, которые являются слоями симплектического слоения $\mathcal{G}^{*}$.

ЗАмЕЧАниЕ 5. Пусть гамильтоновы векторные поля $\vartheta_{i}$ производящих функций $F_{i}$, которые образуют алгебру Ли $\mathcal{G}$, являются полными. Тогда они определяют локально свободное гамильтоново действие в $Z$ некоторой связной группы Ли $G$, алгебра Ли которой изоморфна $\mathcal{G}$. Орбиты $G$ совпадают с $k$-мерными максимальными интегральными многообразиями регулярного распределения $\mathcal{V}$ на $Z$, образованного гамильтоновыми векторными полями $\vartheta_{i}[18]$. Пусть $z \in Z-$ некоторая точка, $G_{z}-$ орбита группы $G$ через $z$. Тогда $\widehat{F}(3)$ отображает эту орбиту на орбиту $G_{F(z)}$ через точку $\widehat{F}(z)$ коприсоединенного действия $\mathcal{G}$ в $\mathcal{G}^{*}$, причем согласно пункту 2 леммы 2 прообраз $\widehat{F}^{-1}\left(G_{F(z)}\right)$ совпадает с орбитой $G_{z}$. Таким образом, орбиты $G$ в $Z$ являются расслоенными многообразиями со слоями - компонентами связности слоев расслоенного многообразия $\widehat{F}(3)$. 
Теорема 8. Пусть $U$ - открытое подмножество $\mathcal{G}^{*}$ такое, что выполняются условия 1 и 2 теоремы 4. Тогда открытое подмножество $\widehat{F}^{-1}(U) \subset Z$ имеет обобщенные координаты действие-угол и система $\left\{F_{1}, \ldots, F_{k}\right\}$ является глобально суперинтегрируемой на $\widehat{F}^{-1}(U)$.

Следующие два примера глобально суперинтегрируемых систем существенно используются в дальнейшем при анализе задачи Кеплера (раздел 7).

Пример 1. Рассмотрим суперинтегрируемую систему $F=\left(F_{1}, F_{2}, F_{3}\right)$ на четырехмерном симплектическом многообразии $Z$, производящие функции которой образуют алгебру Ли $s o(3)$ коранга 1 с коммутационными соотношениями

$$
\left\{F_{1}, F_{2}\right\}=F_{3}, \quad\left\{F_{2}, F_{3}\right\}=F_{1}, \quad\left\{F_{3}, F_{1}\right\}=F_{2} .
$$

Расслоенное многообразие (3)

$$
\widehat{F}: Z \rightarrow N \subset \mathcal{G}^{*}
$$

является отображением моментов многообразия $Z$ в коалгебру Ли $\mathcal{G}^{*}=s o(3)^{*}$, наделенную координатами $\left(x_{i}\right)$ такими, что $F_{i}=x_{i} \circ \widehat{F}$. Коиндуцированная структура Ли-Пуассона коранга 1 на $N$ задана бивекторным полем

$$
w=x_{2} \partial^{3} \wedge \partial^{1}+x_{3} \partial^{1} \wedge \partial^{2}+x_{1} \partial^{2} \wedge \partial^{3} .
$$

Ее функции Казимира имеют вид

$$
C=x_{1}^{2}+x_{2}^{2}+x_{3}^{2}
$$

Пуассоново многообразие $N$ в (13) может быть наделено координатами $\left(I, x_{1}, \gamma\right)$, определенными равенствами

$$
I=x_{1}^{2}+x_{2}^{2}+x_{3}^{2}, \quad x_{2}=\left(I-x_{1}^{2}\right)^{1 / 2} \sin \gamma, \quad x_{3}=\left(I-x_{1}^{2}\right)^{1 / 2} \cos \gamma .
$$

Легко заметить, что координаты (16) - это координаты Дарбу структуры Ли-Пуассона (14) на $N$, а именно

$$
w=\frac{\partial}{\partial x_{1}} \wedge \frac{\partial}{\partial \gamma}
$$

Пусть гамильтоновы векторные поля $\vartheta_{i}$ производящих функций $F_{i}$ полны и слои расслоенного многообразия $\widehat{F}(13)$ связны. Тогда группа $G$ потоков этих векторных полей компактна и ее орбиты в $Z$ компактны. Поскольку компактное расслоенное многообразие является расслоением с компактными слоями (теорема 5), мы получаем, что одномерные слои расслоенного многообразия $\widehat{F}(13)$ компактны, т. е. представляют собой окружности $S^{1}$ (замечание 5). Таким образом, выполняется теорема 8 , и система $F(12)$ является глобально суперинтегрируемой. Пусть $\widehat{F}^{*} C-$ функция на $Z$, индуцированная функцией Казимира $C=I(15)$. Согласно пункту 2 теоремы 6 ее гамильтоново векторное поле полно и его интегральные многообразия слои расслоения (13). Пусть $\alpha$ - параметр вдоль потока этого векторного поля, т. е. $\vartheta_{I}=\partial / \partial \alpha$. Тогда $Z$ имеет обобщенные координаты действие-угол $\left(I, \alpha, x_{1}, \gamma\right)$ такие, что пуассоново бивекторное поле на $Z$ принимает форму

$$
W=\frac{\partial}{\partial I} \wedge \frac{\partial}{\partial \alpha}+\frac{\partial}{\partial x_{1}} \wedge \frac{\partial}{\partial \gamma}
$$


Пример 2. Рассмотрим суперинтегрируемую систему $F=\left(F_{1}, F_{2}, F_{3}\right)$ на четырехмерном симплектическом многообразии $Z$, производящие функции которой образуют алгебру Ли $s o(2,1)$ коранга 1 с коммутационными соотношениями

$$
\left\{F_{1}, F_{2}\right\}=-F_{3}, \quad\left\{F_{2}, F_{3}\right\}=F_{1}, \quad\left\{F_{3}, F_{1}\right\}=F_{2} .
$$

Расслоенное многообразие (3)

$$
\widehat{F}: Z \rightarrow N \subset \mathcal{G}^{*}
$$

является отображением моментов многообразия $Z$ в коалгебру Ли $\mathcal{G}^{*}=s o(2,1)^{*}$, наделенную координатами $\left(x_{i}\right)$ такими, что $F_{i}=x_{i} \circ \widehat{F}$. Коиндуцированная структура Ли-Пуассона коранга 1 в $N$ задана бивекторным полем

$$
w=x_{2} \partial^{3} \wedge \partial^{1}-x_{3} \partial^{1} \wedge \partial^{2}+x_{1} \partial^{2} \wedge \partial^{3} .
$$

Орбиты коприсоединенного представления (11) алгебры Ли $s o(2,1)$ на многообразии $N(19)$ имеет вид

$$
x_{1}^{2}+x_{2}^{2}-x_{3}^{2}=\text { const. }
$$

Это поверхности уровня функции Казимира

$$
C=x_{1}^{2}+x_{2}^{2}-x_{3}^{2}
$$

структуры Пуассона (20). Пуассоново многообразие $N$ (19) может быть наделено координатами $\left(I, x_{1}, \lambda\right)$, которые определяются равенствами

$$
I=x_{1}^{2}+x_{2}^{2}-x_{3}^{2}, \quad x_{2}=\left(I-x_{1}^{2}\right)^{1 / 2} \operatorname{ch} \lambda, \quad x_{3}=\left(I-x_{1}^{2}\right)^{1 / 2} \operatorname{sh} \lambda .
$$

Координаты (23) - это координаты Дарбу структуры Ли-Пуассона (20) на многообразии $N$, а именно

$$
w=\frac{\partial}{\partial \lambda} \wedge \frac{\partial}{\partial x_{1}} .
$$

Пусть гамильтоновы векторные поля $\vartheta_{i}$ производящих функций $F_{i}$ полны и слои расслоенного многообразия $\widehat{F}(19)$ связны. Пусть $G$ - группа потоков этих векторных полей. Ее орбиты представляют собой расслоенные многообразия над орбитами (21) коприсоединенного представления с некомпактными одномерными слоями $\mathbb{R}$, которые совпадают со слоями расслоенного многообразия $\widehat{F}$ (19) (замечание 5). Такое расслоенное многообразие является расслоением (теорема 5). Таким образом, выполняется теорема 8, и система $F(18)$ является глобально суперинтегрируемой. Пусть $\widehat{F}^{*} C$ - функция на $Z$, индуцированная функцией Казимира $C=I(22)$. Согласно пункту 2 теоремы 6 ее гамильтоново векторное поле полно и его интегральные многообразия - слои расслоения (19). Пусть $\tau$ - параметр вдоль потока этого векторного поля, т. е. $\vartheta_{I}=\partial / \partial \tau$. Тогда $Z$ имеет обобщенные координаты действие-угол $\left(I, \lambda, x_{1}, \tau\right)$ такие, что пуассоново бивекторное поле на $Z$ принимает форму

$$
W=\frac{\partial}{\partial I} \wedge \frac{\partial}{\partial \tau}+\frac{\partial}{\partial \lambda} \wedge \frac{\partial}{\partial x_{1}} .
$$




\section{6. ДВИЖЕНИЕ В ЦЕНТРАЛЬНО-СИММЕТРИЧНОМ ПОЛЕ}

Характерным примером композиции глобально суперинтегрируемых систем как с компактными, так и некомпактными инвариантными подмногообразиями служит движение в центрально-симметричном поле.

Рассмотрим гамильтоновы системы в центрально-симметричном поле на фазовом пространстве $\mathbb{R}^{2 n}$ с декартовыми координатами $\left(q^{i}, p_{i}\right), i=1, \ldots, n$, и симплектической формой

$$
\Omega=d p_{i} \wedge d q^{i}
$$

Введем обозначения

$$
p=\left(\sum_{i}\left(p_{i}\right)^{2}\right)^{1 / 2}, \quad r=\left(\sum_{i}\left(q^{i}\right)^{2}\right)^{1 / 2}, \quad p_{r}=\frac{p_{i} q^{i}}{r}
$$

Гамильтониан такой системы имеет вид

$$
H=\frac{1}{2} p^{2}+V(r)
$$

где $V(r)$ - центрально-симметричный потенциал. Ее интегралами движения, помимо гамильтониана $H$, являются функции момента импульса

$$
M_{i k}=q^{i} p_{k}-q^{k} p_{i}, \quad i, k=1, \ldots, n .
$$

Их скобки Пуассона подчиняются соотношениям

$$
\left\{M_{\mu \nu}, M_{\alpha \beta}\right\}=\eta_{\mu \beta} M_{\nu \alpha}+\eta_{\nu \alpha} M_{\mu \beta}-\eta_{\mu \alpha} M_{\nu \beta}-\eta_{\nu \beta} M_{\mu \alpha}
$$

где $\eta_{\mu \nu}$ - евклидова метрика в пространстве $\mathbb{R}^{n}$. Вид соотношений (29) показывает, что функции момента импульса $M_{i k}(28)$ порождают алгебру Ли $\mathcal{G}=s o(n)$.

Рассмотрим случаи, когда эти функции образуют суперинтегрируемую гамильтонову систему (определения 1 и 4). Это исключено на конфигурационном пространстве размерности $n>4$, когда число генераторов алгебры Ли $s o(n)$ равно или превышает размерность $2 n$ фазового пространства. Это возможно, т. е. выполняется условие 3 определения 1 , при $n=4$ и совместно с гамильтонианом $H$ при $n=2,3$. Исследуем эти варианты.

При $n=4$ коранг алгебры Ли so(4) функций моментов импульса $M_{i k}(28)$, $i, k=1, \ldots, 4$, равен 2 , и она удовлетворяет условию 3 определения 1 . Однако не все эти функции независимы. Можно показать, что в каждой точке открытого плотного подмножества многообразия $\mathbb{R}^{2 n} \backslash\{0\}$ число функционально независимых функций моментов импульса $M_{i k}(28), i, k=1, \ldots, 4$, не превышает $2 n-3$ [19], [20], т. е. в данном случае не превышает пяти, и они не составляют суперинтегрируемую систему.

При $n=3$ коранг алгебры Ли so(3) функций моментов импульса $M_{i k}(28)$, $i, k=1,2,3$, равен 1 . Добавив к ним гамильтониан $H(27)$, мы получаем алгебру Ли $\mathcal{G}$ размерности 4 и коранга 2. Она удовлетворяет условию 3 определения 1. Предположим, что функции моментов импульса $M_{i k}(28), i, k=1,2,3$, и гамильтониан $H(27)$ функционально независимы и их гамильтоновы векторные поля полны 
на некотором открытом подмножестве $Z$ фазового пространства $\mathbb{R}^{6}$. Тем самым они составляют суперинтегрируемую гамильтонову систему на $Z$. В частности, фазовое пространство $Z$ не включает точки с $p=0$ или $r=0$ (см. обозначения $(26)$ ), где функции моментов импульсов $M_{i k}, i, k=1,2,3$, не являются функционально независимыми. Поэтому гамильтониан $H(27)$ на таком пространстве $Z$ можно переписать в виде

$$
H=\frac{p_{r}^{2}}{2}+\frac{M^{2}}{2 r^{2}}+V(r)=\frac{p_{r}^{2}}{2}+V_{\mathrm{eff}}(r),
$$

где

$$
M^{2}=\sum_{i<k} M_{i k}^{2}
$$

- оператор Казимира алгебры Ли so(3). Перейдем далее к обозначениям

$$
F_{1}=M_{13}, \quad F_{2}=M_{23}, \quad F_{3}=-M_{12}, \quad M^{2}=F_{1}^{2}+F_{2}^{2}+F_{3}^{2}
$$

в примере 1. Суперинтегрируемая система $F=\left(H, F_{i}\right)$ определяет расслоенное многообразие $\widehat{F}(13)$ над открытым подмногообразием $N$ коалгебры Ли $\mathcal{G}^{*}=\mathbb{R} \times$ $s o(3)^{*}$ с соответствующими координатами $\left(x_{H}, x_{i}\right)$ и структурой Ли-Пуассона $w(14)$ в данном случае коранга 2. Коприсоединенное представление (11) алгебры Ли $\mathcal{G}$ с генераторами $\left\{\varepsilon, \varepsilon_{i}\right\}$ в $N$ имеет вид

$$
\varepsilon=0, \quad \varepsilon_{1}=x_{3} \partial^{2}-x_{2} \partial^{3}, \quad \varepsilon_{2}=x_{1} \partial^{3}-x_{3} \partial^{1}, \quad \varepsilon_{3}=x_{2} \partial^{1}-x_{1} \partial^{2} .
$$

Орбиты этого коприсоединенного представления задаются уравнениями

$$
x_{H}=\text { const }, \quad x_{1}^{2}+x_{2}^{2}+x_{3}^{2}=\text { const. }
$$

Это поверхности уровня функций Казимира

$$
C_{H}=x_{H}, \quad C_{M}=x_{1}^{2}+x_{2}^{2}+x_{3}^{2}
$$

структуры Ли-Пуассона $w$. Пуассоново многообразие $N$ может быть наделено координатами $\left(x_{H}, I, x_{1}, \gamma\right)$, которые определяются равенствами (16). В этих координатах структура Ли-Пуассона $w$ принимает вид (17). Пусть слои расслоенного многообразия $\widehat{F}(13)$ связны. Следуя замечанию 5 , рассмотрим группу $G$ потоков гамильтоновых векторных полей производящих функций $\left(H, F_{i}\right)$. Расслоенное многообразие $\widehat{F}$ определяет проекцию ее орбит в пространстве $Z$ на орбиты (33) коприсоединенного представления в $N$, слоями которой являются инвариантные подмногообразия суперинтегрируемой гамильтоновой системы $\left(H, F_{i}\right)$, представляющие собой максимальные интегральные многообразия гамильтоновых векторных полей функций $H=\widehat{F}^{*} C_{H}$ и $M^{2}=\widehat{F}^{*} C_{M}$ (лемма 2). Имея своей алгеброй Ли $\mathcal{G}^{*}$, группа Ли $G$ может быть как компактна (изоморфна $U(1) \times S O(3)$ ), так и некомпактна (изоморфна $\mathbb{R} \times S O(3)$ ). В первом случае, на основании тех же аргументов, что и в примере 1 , слои расслоенного многообразия $\widehat{F}$ компактны и диффеоморфны тору $T^{2}$. Во втором случае это некомпактные цилиндры $\mathbb{R} \times S^{1}$. Различие обусловлено тем, какую именно группу $(U(1)$ или $\mathbb{R})$ порождает поток гамильтонова векторного поля гамильтониана $H=\widehat{F}^{*} C_{H}$. 
При $n=2$ движение в центрально-симметричном поле общего вида имеет два интеграла движения - гамильтониан $H(27)$ и функцию момента импульса

$$
M=M_{12}=q^{1} p_{2}-q^{2} p_{1}
$$

Предположим, что они независимы и их гамильтоновы векторные поля полны на некотором открытом подмножестве $Z$ фазового пространства $\mathbb{R}^{4}$. Тем самым они составляют вполне интегрируемую гамильтонову систему на $Z$. В частности, фазовое пространство $Z$ не включает точки с $p=r=0$, где функция момента импульса $M(35)$ нерегулярна. Вполне интегрируемая система $F=(H, M)$ определяет расслоенное многообразие $\widehat{F}(13)$ :

$$
\widehat{F}=(H, M): P \rightarrow N \subset \mathbb{R}^{4},
$$

база которого $N$ наделяется координатами $\left(x_{H}, x_{M}\right)$ такими, что $x_{H} \circ \widehat{F}=H, x_{M} \circ$ $\widehat{F}=M$. Поскольку поток гамильтонова векторного поля

$$
\vartheta_{M}=q^{1} \partial_{2}-q^{2} \partial_{1}+p_{1} \partial^{2}-p_{2} \partial^{1}
$$

функции момента импульса $M(35)$ порождает компактную группу, слои расслоенного многообразия $\widehat{F}(36)$ или компактны и диффеоморфны тору $T^{2}$, или некомпактны и диффеоморфны цилиндру $\mathbb{R} \times S^{1}$. Над теми открытыми подмногообразиями $U \subset N$, где расслоенное многообразие $\widehat{F}(36)$ является расслоением со связными слоями, вполне интегрируемая гамильтонова система $(H, M)$ глобально интегрируема (теорема 4).

ПримеР 3. Рассмотрим задачу Кеплера с гамильтонианом

$$
H=\frac{1}{2} p^{2}-\frac{1}{r}
$$

на фазовом пространстве $\mathbb{R}^{4} \backslash\{r=0\}$. На этом пространстве можно ввести систему полярных координат и сопряженных канонических импульсов

$$
q^{1}=r \cos \alpha, \quad q^{2}=r \sin \alpha, \quad p_{\alpha}=M, \quad p_{r}=p_{1} \cos \alpha+p_{2} \sin \alpha .
$$

В этих координатах функция момента импульса $M(35)$ и гамильтониан $H(37)$ принимают вид

$$
M=p_{\alpha}, \quad H=\frac{p_{r}^{2}}{2}+\frac{p_{\alpha}^{2}}{2 r^{2}}-\frac{1}{r}=\frac{p_{r}^{2}}{2}+V_{\mathrm{eff}}(r) .
$$

Они функционально зависимы в точках, где

$$
p_{r}=0, \quad \partial_{r} V_{\mathrm{eff}}=\left(-\frac{p_{\alpha}^{2}}{2 r^{3}}+\frac{1}{r^{2}}\right)=0,
$$

и образуют вполне интегрируемую гамильтонову систему на фазовом пространстве

$$
Z=\mathbb{R}^{4} \backslash\{r=0\} \backslash\left\{p_{r}=0, p_{\alpha}^{2}=r\right\} \backslash\left\{p_{\alpha}=0\right\}
$$


Интегралы движения $F=(H, M)(38)$ формируют на нем вполне интегрируемую гамильтонову систему и определяют расслоенное многообразие

$$
\widehat{F}: Z \rightarrow N \subset \mathbb{R}^{2}
$$

база $N$ которого наделена координатами $\left(x_{H}, p_{\alpha}\right)$ и состоит из двух непересекающихся открытых областей с координатами $p_{\alpha}<0$ и $p_{\alpha}>0$. Слои расслоенного многообразия (41) задаются в фазовом пространстве $Z$ (40) уравнениями $H=$ const, $p_{\alpha}=$ const, но они не взаимно диффеоморфны. Следуя анализу в случае $n=3$, можно показать, что при $x_{H}<0$ это торы $T^{2}$, а при $x_{H} \geqslant 0$ - цилиндры $\mathbb{R} \times S^{1}$. В результате фазовое пространство $Z$ (41) включает четыре непересекающиеся области $\left(x_{H}<0, p_{\alpha}<0\right),\left(x_{H}<0, p_{\alpha}>0\right),\left(x_{H}>0, p_{\alpha}<0\right)$ и $\left(x_{H}>0, p_{\alpha}>0\right)$, где расслоенное многообразие $\widehat{F}$ является расслоением, а вполне интегрируемая гамильтонова система $(H, M)(38)$ - глобально интегрируемой, причем с компактными инвариантными подмногообразиями в первых двух областях и некомпактными в последних двух. Однако, как известно, интегралы движения в двумерной задаче Кеплера не исчерпываются функцией момента импульса, а представлены еще двумя компонентами $A_{i}, i=1,2$, вектора Рунге-Ленца (см. ниже $\left.(42)\right)$. Функции $\left(M, A_{i}\right)$ независимы на некотором открытом подмногообразии фазового пространства и порождают на нем некоммутативную суперинтегрируемую систему коранга 1 с алгеброй Ли so(3) или $s o(2,1)$ (раздел 7$)$. В отличие от вполне интегрируемой гамильтоновой системы $(H, M)(38)$, ее инвариантные подмногообразия одномерны, что дает более точный анализ задачи Кеплера. Кроме того, фазовое пространство $Z$ (40) вполне интегрируемой системы $(H, M)(38)$ не включает траектории движения по окружности (39). Поэтому в следующем разделе мы обратимся к анализу суперинтегрируемой системы $\left(M, A_{i}\right)$ задачи Кеплера.

\section{7. ЗАДАЧА КЕПЛЕРА}

Задача Кеплера на фазовом симплектическом многообразии $\mathbb{R}^{2 n} \backslash\{r=0\}$ с потенциалом $V(r)=-\kappa r^{-1}, \kappa= \pm 1$, наряду с $M_{i k}(28)$, имеет еще интегралы движения

$$
A_{i}=\sum_{j} M_{i j} p_{j}-\frac{q^{i}}{r}=q^{i} p^{2}-p_{i}(p, q)-\frac{q^{i}}{r},
$$

называемые вектором Рунге-Ленца. Они удовлетворяют соотношениям

$$
\left\{M_{i j}, A_{k}\right\}=\eta_{j k} A_{i}-\eta_{i k} A_{j}, \quad\left\{A_{i}, A_{j}\right\}=2 H M_{i j},
$$

где $H$ - гамильтониан задачи Кеплера. Общее число интегралов движения $M_{i k}(28)$ и $A_{i}(42)$ меньше размерности $2 n$ только при $n=2$, и только в этом случае они могут составить суперинтегрируемую систему.

Поэтому рассмотрим задачу Кеплера в двумерном конфигурационном пространстве $\mathbb{R}^{2} \backslash\{0\}$ как с притягивающим $(\kappa=1)$, так и с отталкивающим $(\kappa=-1)$ потенциалом. Ее гамильтониан на фазовом симплектическом многообразии $\mathbb{R}^{4} \backslash\{r=0\}$ имеет вид

$$
H=\frac{1}{2} p^{2}-\frac{\kappa}{r}, \quad \kappa= \pm 1
$$


В отличие от примера 3, в данном случае мы характеризуем задачу интегралами движения

$$
M=M_{12}=q^{1} p_{2}-q^{2} p_{1}, \quad A_{1}=M p_{2}-q^{1} \frac{\kappa}{r}, \quad A_{2}=-M p_{1}-q^{2} \frac{\kappa}{r},
$$

которые функционально независимы на фазовом пространстве

$$
Z=\mathbb{R}^{4} \backslash\{r=0\} \backslash\left\{p^{2}=0\right\} \backslash\{M=0\} .
$$

Гамильтониан $H$ (14) регулярен и функционально зависим с интегралами движения $\left(M, A_{1}, A_{2}\right)(45)$ на фазовом пространстве $Z(46)$, так что выполняется соотношение

$$
H=\frac{A_{1}^{2}+A_{2}^{2}-1}{2 M^{2}} .
$$

Хорошо известная динамика задачи Кеплера также свидетельствует, что гамильтоново векторное поле гамильтониана $H$ на фазовом пространстве $Z$ (46) является полным.

Скобки Пуассона (43) интегралов движения $\left(M, A_{1}, A_{2}\right)(45)$ принимают вид

$$
\begin{gathered}
\left\{M, A_{1}\right\}=-A_{2}, \quad\left\{M, A_{2}\right\}=A_{1}, \\
\left\{A_{1}, A_{2}\right\}=2 H M=\frac{A_{1}^{2}+A_{2}^{2}-1}{M} .
\end{gathered}
$$

Эти соотношения имеют форму (4). Однако коранг матричной функции s коммутационных соотношений (48), (49) не постоянен в точках, для которых $H=0$. Поэтому рассмотрим открытые подмногообразия $U_{-} \subset Z$, где $H<0$, и $U_{+}$, где $H>0$. Тогда задачи Кеплера с гамильтонианом $H(44)$ и интегралами движения $\left(M, A_{1}, A_{2}\right)$ (45) на $U_{-}$и на $U_{+}$являются суперинтегрируемыми гамильтоновыми системами. Более того, эти суперинтегрируемые системы могут быть приведены к форме (9) следующим образом.

Заменим интегралы движения $A_{i}$ на $U_{-}$интегралами движения

$$
L_{i}=\frac{A_{i}}{\sqrt{-2 H}}
$$

и на $U_{+}$интегралами движения

$$
K_{i}=\frac{A_{i}}{\sqrt{2 H}}
$$

Суперинтегрируемая система $\left(M, L_{i}\right)$ на $U_{-}$подчиняется соотношениям

$$
\left\{M, L_{1}\right\}=-L_{2}, \quad\left\{M, L_{2}\right\}=L_{1}, \quad\left\{L_{1}, L_{2}\right\}=-M,
$$

а равенство (47) принимает вид

$$
M^{2}+L^{2}=-\frac{1}{2 H} .
$$

Обозначим $M_{i 3}=-L_{i}$ и введем индексы $\mu, \nu, \alpha, \beta=1,2,3$. Тогда соотношения (52) приводятся к коммутационным соотношениям (29) алгебры Ли $\mathcal{G}=s o(3)$. 
Суперинтегрируемая система $\left(M, K_{i}\right)$ на $U_{+}$подчиняется соотношениям

$$
\left\{M, K_{1}\right\}=-K_{2}, \quad\left\{M, K_{2}\right\}=K_{1}, \quad\left\{K_{1}, K_{2}\right\}=-M,
$$

а равенство (47) принимает форму

$$
K^{2}-M^{2}=\frac{1}{2 H}
$$

Обозначим $M_{i 3}=-K_{i}$ и введем индексы $\mu, \nu, \alpha, \beta=1,2,3$. Тогда соотношения (54) приводятся к виду

$$
\left\{M_{\mu \nu}, M_{\alpha \beta}\right\}=\rho_{\mu \beta} M_{\nu \alpha}+\rho_{\nu \alpha} M_{\mu \beta}-\rho_{\mu \alpha} M_{\nu \beta}-\rho_{\nu \beta} M_{\mu \alpha},
$$

где $\rho_{\mu \nu}$ - псевдоевклидова метрика сигнатуры $(+,+,-)$ на $\mathbb{R}^{3}$. Это коммутационные соотношения алгебры Ли so $(2,1)$ коранга 1.

Таким образом, задача Кеплера на фазовом пространстве $Z$ (46) распадается на две различные суперинтегрируемые гамильтоновы системы на открытых подмногообразиях $U_{-}$и $U_{+}$, причем в случае отталкивающего потенциала $(\kappa=-1)$ всегда $H>0$ и $U_{+}$совпадает со всем фазовым пространством $Z(46)$.

Обратимся к первой из этих систем. Приведем ее к виду, рассматривавшемуся в примере 1 , положив $F_{1}=-L_{1}, F_{2}=-L_{2}, F_{3}=-M$. Заметим, однако, что в данном случае база $N$ расслоенного многообразия (13) - это несвязное многообразие, являющееся объединением двух связных компонент с координатами $x_{3}>0$ и $x_{3}<0$. Рассмотрим функцию Казимира $C(15)$ и получаемую из нее функцию Казимира

$$
h=-\frac{1}{2 C}=-\frac{1}{2}\left(x_{1}^{2}+x_{2}^{2}+x_{3}^{2}\right)^{-1} .
$$

Вид соотношения (53) показывает, что функция $\widehat{F}^{*} h$, индуцированная на $U_{-}$функцией Казимира (57), является гамильтонианом $H(44)$ при $\kappa=1$ задачи Кеплера на $U_{-}$. Как было отмечено выше, гамильтоново векторное поле функции $\widehat{F}^{*} h$ полно. Поэтому, следуя рассуждениям в примере 1 , мы получаем, что задача Кеплера на $U_{-}$является глобально суперинтегрируемой гамильтоновой системой с компактными инвариантными подмногообразиями $S^{1}$ и тем самым допускает глобальные обобщенные переменные действие-угол. Пуассоново многообразие $N$ может быть наделено координатами $\left(J, x_{1}, \gamma\right)$, где координата $J$, в отличие от координаты $I(16)$ в примере 1 , задана равенством

$$
J=-\frac{1}{2 I}=-\frac{1}{2}\left(x_{1}^{2}+x_{2}^{2}+x_{3}^{2}\right)^{-1} .
$$

Пусть $\vartheta_{J}$ - гамильтоново векторное поле функции $\widehat{F}^{*} h=J$, а $\lambda$ - параметр вдоль потока этого векторного поля, т. е.

$$
\vartheta_{J}=\frac{\partial}{\partial \lambda}
$$

Тогда $N$ наделяется обобщенными координатами действие-угол $\left(J, x_{1}, \gamma, \lambda\right)$. При этом векторное поле $\vartheta_{J}(58)$ имеет вид

$$
\frac{\partial}{\partial \lambda}=\sum_{i}\left(\frac{\partial H}{\partial p_{i}} \frac{\partial}{\partial q_{i}}-\frac{\partial H}{\partial q_{i}} \frac{\partial}{\partial p_{i}}\right) .
$$


Это равенство приводит к соотношениям типа уравнений Гамильтона:

$$
\frac{\partial q_{i}}{\partial \lambda}=\frac{\partial H}{\partial p_{i}}, \quad \frac{\partial p_{i}}{\partial \lambda}=-\frac{\partial H}{\partial q_{i}} .
$$

Поэтому обобщенная угловая координата $\lambda$ - это циклическое время $\lambda=t \bmod (2 \pi)$, которое дается известными выражениями

$$
\begin{aligned}
& \lambda=\phi-a^{3 / 2} e \sin \left(a^{-3 / 2} \phi\right), \quad r=a\left(1-e \cos \left(a^{-3 / 2} \phi\right)\right), \\
& a=-\frac{1}{2 J}, \quad e=\left(1+2 J M^{2}\right)^{1 / 2} .
\end{aligned}
$$

Рассмотрим теперь задачу Кеплера на $U_{+}$при $H>0$. Приведем ее к виду, рассматривавшемуся в примере 2 , положив $F_{1}=-K_{1}, F_{2}=-K_{2}, F_{3}=-M$. Рассмотрим функцию Казимира $C(22)$ и получаемую из нее функцию Казимира

$$
h=-\frac{1}{2 C}=\frac{1}{2}\left(x_{1}^{2}+x_{2}^{2}-x_{3}^{2}\right)^{-1} .
$$

Вид соотношения (55) показывает, что функция $\widehat{F}^{*} h$, индуцированная на $U_{+}$функцией Казимира (59), является гамильтонианом $H(44)$ задачи Кеплера на $U_{+}$. Как было отмечено выше, гамильтоново векторное поле функции $\widehat{F}^{*} h$ полно. Поэтому, следуя рассуждениям в примере 2 , мы получаем, что задача Кеплера на $U_{+}$ является глобально суперинтегрируемой гамильтоновой системой с инвариантными многообразиями $\mathbb{R}$ и тем самым допускает глобальные обобщенные переменные действие-угол. Пуассоново многообразие $N$ может быть наделено координатами $\left(J, x_{1}, \gamma\right)$, где координата $J$, в отличие от координаты $I(23)$ в примере 2, задана равенством

$$
J=\frac{1}{2 I}=\frac{1}{2}\left(x_{1}^{2}+x_{2}^{2}-x_{3}^{2}\right)^{-1} .
$$

Пусть $\vartheta_{J}$ - гамильтоново векторное поле функции $\widehat{F}^{*} h=J$, а $\tau$ - параметр вдоль потока этого векторного поля, т. е.

$$
\vartheta_{J}=\frac{\partial}{\partial \tau} .
$$

Тогда $N$ имеет обобщенные координаты действие-угол $\left(J, x_{1}, \gamma, \lambda\right)$. Так же, как и обобщенная угловая переменная $\lambda(58)$, обобщенная угловая переменная $\tau$ (60) удовлетворяет уравнениям Гамильтона

$$
\frac{\partial q_{i}}{\partial \tau}=\frac{\partial H}{\partial p_{i}}, \quad \frac{\partial p_{i}}{\partial \tau}=-\frac{\partial H}{\partial q_{i}},
$$

поэтому она является переменной времени $\tau=t$, задаваемой известными выражениями

$$
\begin{aligned}
& \tau=s-a^{3 / 2} e \operatorname{sh}\left(a^{-3 / 2} s\right), \quad r=a\left(e \operatorname{ch}\left(a^{-3 / 2} s\right)-1\right), \\
& a=\frac{1}{2 J}, \quad e=\left(1+2 J M^{2}\right)^{1 / 2} .
\end{aligned}
$$

Отметим, что глобально суперинтегрируемые системы на $U_{-}$при $H<0$ и $U_{+}$ при $H>0$ не описывают движение по параболе при $H=0$. Его инвариантное подмногообразие некомпактно, однако всякая насыщенная окрестность этого подмногообразия содержит компактные инвариантные подмногообразия, и они вместе не образуют расслоения. 


\section{8. ЗАКЛЮЧЕНИЕ}

Представленные в работе теоремы 1, 4, 6 и 7 обобщают теорему Мищенко-Фоменко (теорема 2) на общий случай суперинтегрируемых гамильтоновых систем как с компактными (торы $T^{m}$ ), так и с некомпактными (тороидальные цилиндры $\mathbb{R}^{m-r} \times T^{r}$ ) инвариантными подмногообразиями и тем самым позволяют проводить глобальный анализ таких систем. Это важно, поскольку суперинтегрируемая система в общем случае представляет собой композицию нескольких неэквивалентных глобально суперинтегрируемых систем (с неизоморфными алгебрами производящих функций и недиффеоморфными инвариантными подмногообразиями) на разных непересекающихся открытых подпространствах фазового симплектического многообразия.

В разделах 5-7 исследован ряд характерных примеров таких суперинтегрируемых систем с некомпактными инвариантными подмногообразиями. В частности, их анализ показывает, что условие 3 в определении 1 является весьма ограничивающим. Его возможно ослабить, перейдя к суперинтегрируемым системам на пуассоновых многообразиях [21].

\section{Список литературы}

[1] А. С. Мищенко, А. Т. Фоменко, “Обобщенный метод Лиувилля интегрирования гамильтоновых систем", Функи. анализ и его прил., 12:2 (1978), 46-56.

[2] A. V. Bolsinov, B. Jovanović, "Noncommutative integrability, moment map and geodesic flows", Ann. Global Anal. Geom., 23:4 (2003), 305-322.

[3] F. Fassò, "Francesco Superintegrable Hamiltonian systems: geometry and perturbations", Acta Appl. Math., 87:1-3 (2005), 93-121.

[4] V. I. Arnold (ed.), Dynamical Systems III, IV, Springer, Berlin, 1990.

[5] V.F. Lazutkin, KAM Theory and Semiclassical Approximations to Eigenfunctions, Ergebnisse der Mathematik und ihrer Grenzgebiete, 24, Springer, Berlin, 1993.

[6] G. Giachetta, L. Mangiarotti, G. Sardanashvily, "Bi-Hamiltonian partially integrable systems", J. Math. Phys., 44:5 (2003), 1984-1997.

[7] E. Fiorani, G. Giachetta, G. Sardanashvily, "The Liouville-Arnold-Nekhoroshev theorem for non-compact invariant manifolds", J. Phys. A, 36:7 (2003), L101-L107.

[8] E. Fiorani, G. Sardanashvily, "Noncommutative integrability on noncompact invariant manifolds", J. Phys. A, 39:45 (2006), 14035-14042.

[9] G. Sardanashvily, "Superintegrable Hamiltonian systems with noncompact invariant submanifolds: Kepler system", Internat. J. Geom. Methods Modern Phys., 6:8 (2009), 1391-1414.

[10] G. Sardanashvily, Handbook of Integrable Hamiltonian Systems, URSS, Moscow, 2015.

[11] E. Fiorani, G. Sardanashvily, "Global action-angle coordinates for completely integrable systems with noncompact invariant submanifolds", J. Math. Phys., 48:3 (2007), 032901, $9 \mathrm{pp}$.

[12] J. Duistermaat, "On global action-angle coordinates", Commun. Pure Appl. Math., 33:6 (1980), 687-706.

[13] P. Dazord, T. Delzant, "Le probleme general des variables actions-angles", J. Differ. Geom., 26:2 (1987), 223-251.

[14] I. Vaisman, Lectures on the Geometry of Poisson Manifolds, Progress in Mathematics, 118, Birkhäuser, Basel, 1994.

[15] R. S. Palais, A Global Formulation of the Lie Theory of Transformation Groups, Memoirs of the American Mathematical Society, 22, AMS, Providence, RI, 1957. 
[16] G. Meigniez, "Submersions, fibrations and bundles", Trans. Amer. Math. Soc., 354:9 (2002), 3771-3787.

[17] V. Guillemin, S. Sternberg, Symplectic Techniques in Physics, Cambridge Univ. Press, Cambridge, 1984.

[18] H. J. Sussmann, "Orbits of families of vector fields and integrability of distributions", Trans. Amer. Math. Soc., 180 (1973), 171-188.

[19] R.H. Cushman, L. M. Bates, Global Aspects of Classical Integrable Systems, Birkhäuser, Basel, 1997.

[20] E. Fiorani, "Momentum maps, independent first integrals and integrability for central potentials", Internat. J. Geom. Methods Modern Phys., 6:8 (2009), 1323-1341.

[21] A. Kurov, G. Sardanashvily, Partially superintegrable systems on Poisson manifolds, arXiv: 1606.03868 .

Поступила в редакцию 12.05.2016, после доработки 20.06.2016 